\title{
Disability among elderly rural villagers: report of a survey from Gonoshasthaya Kendra, Bangladesh
}

\author{
Nicola Cherry ${ }^{1 *}$, Morshed Chowdhury ${ }^{2}$, Rezaul Haque ${ }^{3}$, Corbett McDonald ${ }^{4}$ and Zafrullah Chowdhury ${ }^{5}$
}

\begin{abstract}
Background: The study was set up to identify the extent and nature of difficulty with activities of daily living (disabilities) among elderly village residents of Bangladesh, to describe help currently given and to identify possible interventions. It was carried out at Gonoshasthaya Kendra (GK), a community development organization responsible for the health care of 600 villages with a population of some 1.5 million.

Methods: A survey card was designed and piloted using 12 questions on disability, elaborated from the Washington Group Disability questions, together with a checklist of health problems. A survey was carried out in 2010 in 535 villages under the care of GK since 2005, with village paramedics interviewing residents believed to be age 60 years or older. Respondents were matched where possible to data from the 2005 GK household census, giving data on education, occupation, socioeconomic group and smoking habit.

Results: Survey cards were completed for 43417 residents of which 17346 were matched to residents recorded in the GK census as born $\leq 1945$. The proportion reporting 'much difficulty' on one or more functional capacities increased steadily with age, reaching 55\% (1796/3620) among those $\geq 85$ years. Difficulties most frequently reported were lifting and carrying, vision and going outside the home. At all ages women were more likely to report 'much difficulty' than men $(\mathrm{OR}=1.43(1.35$ to 1.48$))$, with widows and the illiterate at greater risk. Health problems, particularly hemiplegia, resting tremor, urinary incontinence and depression were strongly related to the 12 disabilities assessed. Help came almost entirely from family members; of 11211 villagers with 'much difficult' on at least one functional capacity, only 15 reported getting help outside the family.
\end{abstract}

Conclusions: Disabled elderly residents were dependent on the family for help but, with family cohesiveness under threat from migration to the city, there is a pressing need for the development and critical evaluation of community-based interventions designed specifically for the elderly in poor rural societies. New approaches to training and practice will be needed to integrate such disability management into primary care.

\section{Background}

Bangladesh is a poor, largely rural, country with a population of more than 150 million. Although health services remain limited, much has been achieved among the young, but with little care from outside the family for the growing population of the rural elderly. The present study was designed to identify important difficulties in functional capacity in the elderly living in villages under the care of Gonoshasthaya Kendra (GK), a community development organization which provides primary health care through paramedics trained for

\footnotetext{
* Correspondence: nicola.cherry@ualberta.ca

${ }^{1}$ University of Alberta, 5-30 University Terrace, 8303-112 St, Edmonton, AB,

Canada T6G 1 K4

Full list of author information is available at the end of the article
}

2 years within GK. At the time of the survey GK was responsible for the health care of 600 villages with a population of some 1.5 million. Rural health care was administered through 16 sub-centres, administering 40 health centres, from which a paramedic was assigned responsibility for each village, providing front line care.

Our aim was to collect information that would serve to improve the management of disability in the elderly. The WHO Study on global AGEing and adult heath (SAGE) has developed interview schedules to collect data on ageing, which have been used in some less developed countries including one area of Bangladesh [1], but the schedules appeared too complex for our goal of identifying interventions that might be helpful in poor rural communities. We adopted instead the set of 6

\section{Biomed Central}


disability questions developed by the 'Washington Group ', covering vision, hearing, remembering or concentrating, walking or climbing stairs, self care (washing or dressing) and communicating [2]. We expanded these to help us better understand the circumstances in which difficulties occurred and might be managed. We then used this tool in a survey of elderly villagers living in 535 villages that had been under the care of GK since 2005, when a GK house-to-house census had been conducted by the paramedic assigned to each village.

\section{Methods}

\section{Target population}

1) Conceptual. The survey was designed to include all those still living in 2010 in the village recorded in the 2005 GK census and with a recorded census birth year of 1945 or earlier (i.e. 65 years or greater at the time of the survey).

2) Pragmatic (survey population). All residents of each village believed to be 60 years or older were identified. In the absence of any birth certification true age was difficult to ascertain, either in the census or survey; a standard protocol was used, estimating age from historic events.

3) Matched sub-population. Information from the 2005 census and 2010 survey were matched on household number and sex for all those recorded with a birth before 1945 in the census, thus approximating the initial design.

\section{Survey card}

The survey card (Additional file 1) devised was in 5 parts, demographic (age, sex, marital status, age of living spouse), difficulties with activities of daily life (disability: questions 1-12), health problems (ill-health: Q13), resting tremor, as a coarse screen for Parkinson's disease (Q14) and help received and needed (Q15 and 16). Questions 1 and 2 on the card and the response scale for all disability questions ('no problem' to 'can't do it at all') were taken directly from the Washington Group questions $^{1}$ but the remaining 10 disability questions were elaborated to help identify barriers that might be susceptible to intervention. Respondents were asked to provide their own perception of degree of difficulty but paramedics were asked to record whether this was importantly underrated. The health problems listed were those felt by $\mathrm{GK}$ physicians $(\mathrm{ZC}, \mathrm{RH})$ to be the most troublesome among elderly villagers: space was left to record 'other' problems.

The final English version was translated into Bangla and back-translated before being piloted for comprehension in villages that had come under the care of GK since 2005.

\section{Administration}

Starting in November 2009, the paramedic in each village conducted a house-to-house survey to compile a list of villagers believed to be aged 60 or greater. She then sought to interview all listed, recording the reason for any failure to do so. Supervisors re-interviewed about $10 \%$ of respondents to ensure that the interview had indeed been conducted. Cards were checked for completeness locally and returned to the GK research unit for coding and data entry. Responses to the disability and ill-health questions (Q1-14) were entered as recorded. Up to three responses were coded for open-ended items. Data collection was completed in May 2010.

\section{Matching to census}

Survey respondents were matched to the census data , collected by paramedics in a house-to-house survey in 2005, on village, household number and sex Where a match was achieved, census year of birth, educational level, socioeconomic status (used by GK to determine payment), occupation in 2005 and smoking habit (yes/ no) were added to the survey data file.

\section{Statistical methods}

Three composite scores were calculated, the total number of disabilities coded as either 3 or 4 (range $0-12$ ), a total disability score (the sum of codes $1-4$ on all 12 items: range 12- 48) and the total number of boxes checked (from joints to 'other') at Q13 (range 0-10).

The demographics of the 2 populations (survey and census-matched) were compared and the frequency of reporting each disability and health problem examined by age and sex. Disability was considered to be present only for those reporting that they had either 'much difficulty' (code 3) or 'could not do it at all' (code 4) on Q112. The relation of each disability to age, family structure (living spouse) and census information on poverty, literacy, employment and smoking was examined by logistic regression, stratified by sex. The model also included the health problems listed at Q13 (except prolapse, applicable to women only) and at Q14. In these regressions the effect of each factor (present versus absent) was calculated in a model containing all potential predictors and confounders. The relation of total disability score (range 12-48, log-transformed to reduce skew) to health problems as examined by linear regression. Help received and needed was examined by age, sex and extent of disability.

There were very few missing values on items other than age. In analyses including age missing values were excluded: those reporting an age $<60$ years (but believed to be $\geq 60$ years) were included as a distinct group. The analysis was carried out using SPSS/PASW Statistics 18. 


\section{Results}

\section{Participation and matching}

Survey cards were completed and entered for 43417 residents. Non-completion was recorded for 12969, with 54 recorded as refused, 5821 as died before an interview could be completed, 2006 as having moved out of the village and 1496 as still living there but never found at home. No reason was given for non-completion for 3592. In the absence of an independent nominal roll there was some uncertainty about the true size of the target population, but the response rate estimated from these figures (excluding those who had died or moved away) was $89.4 \%(43417 / 48559)$.

Of the 43417 interviewed, 17346 (40\%) were successfully matched to a villager of the same sex at the same address in the census data of 2005, with a census birth year $\leq 1945$ : scrutiny of the names recorded at the census and survey in a random sample of matched records showed a very high concordance (>95\%). Because of changes in household numbering no matching was possible for 70 villages. Among those in the remaining 465 villages, subjects not matched either did not appear in the census data for that village (as would happen if they had moved into the village since 2005 or had been omitted in error from the census) or appeared in the census but with a different household number or with a birth year $>1945$.

\section{The study populations}

The age and sex reported in the survey are shown in Table 1 for all responders and for the subgroup matched to the census, together with the number of disabilities and health problems reported. The expected deficit (implied by matching on birth $\leq 1945$ ) was evident in the age group $60<65$ years in the census-matched subpopulation. Women were younger than men (were more often $<75$ years) and more likely to be widowed. The proportion with no disability or health problem was very similar in the two populations. Overall nearly three quarters of the respondents reported that they had no serious difficulty with any of the functions listed, but the proportion with difficulties increased steadily with age. In those aged 85 years of greater 55\% (1796/3620) had 'much difficulty' with at least one functional capacity. In this elderly population as a whole $<3 \%$ reported such difficulty on 6 or more capacities, but this rose to $14.6 \%$ (477/3620) in those $\geq 85$ years. Health problems were reported more frequently than 'much difficulty' with functional capacities. Almost all respondents (92.7\%) reported at least one health problem that made life difficult.

At the census (Table 2) some $90 \%$ of these women had been recorded as illiterate as had two thirds of men, with younger respondents being less likely to be illiterate than
Table 1 Distributions of age, sex, marital status, disability and health problems in the survey population and census-matched subpopulation

\begin{tabular}{|c|c|c|c|c|c|c|}
\hline \multicolumn{4}{|c|}{$\begin{array}{l}\text { All survey } \\
\text { respondents } \\
(\mathrm{N}=43417)\end{array}$} & \multicolumn{3}{|c|}{$\begin{array}{l}\text { Respondents } \\
\text { matched to census } \\
(\mathrm{N}=17346)\end{array}$} \\
\hline \multicolumn{2}{|c|}{ Men } & \multicolumn{2}{|c|}{ Women } & \multicolumn{2}{|c|}{ Men } & Women \\
\hline $\mathrm{N}$ & $\%$ & $\mathbf{N}$ & $\%$ & $\mathrm{~N}$ & $\%$ & $\mathbf{N}$ \\
\hline
\end{tabular}

\section{Age from survey}

$\begin{array}{lcccccccc}<60 & 1369 & 6.2 & 1653 & 7.7 & 593 & 6.2 & 670 & 8.5 \\ 60<65 & 3836 & 17.5 & 5102 & 23.7 & 1036 & 10.9 & 1246 & 15.8 \\ 65<75 & 5043 & 23.0 & 5628 & 26.2 & 2261 & 23.7 & 2264 & 28.7 \\ 70<75 & 5033 & 23.0 & 4215 & 19.6 & 2307 & 24.2 & 1707 & 21.6 \\ 75<80 & 2705 & 12.3 & 1672 & 7.8 & 1420 & 14.9 & 743 & 9.4 \\ 80<85 & 1988 & 9.1 & 1608 & 7.5 & 971 & 10.2 & 650 & 8.2 \\ 85<90 & 783 & 3.6 & 524 & 2.4 & 411 & 4.3 & 229 & 2.9 \\ >90 & 1024 & 4.7 & 929 & 4.3 & 483 & 5.1 & 331 & 4.2 \\ \text { Unknown } & 141 & 0.6 & 164 & 0.8 & 55 & 0.6 & 59 & 0.7 \\ \text { TOTAL } & 21922 & 100.0 & 21495 & 100.0 & 9537 & 100.0 & 7899 & 100.0\end{array}$

\section{Living spouse}

$\begin{array}{lcccccccc}\text { No } & 2776 & 12.7 & 12199 & 56.8 & 1119 & 11.7 & 4579 & 58.0 \\ \text { Yes } & 19141 & 87.3 & 9289 & 43.2 & 8416 & 88.2 & 3316 & 42.0 \\ \text { Unknown } & 5 & 0.0 & 7 & 0.0 & 2 & 0.0 & 4 & 0.1 \\ \text { TOTAL } & 21922 & 100.0 & 21495 & 100.0 & 9537 & 100.0 & 7899 & 100.0\end{array}$

Number of disabilities (Q1-12) (much difficulty or worse)

$\begin{array}{lcccccccc}\text { None } & 16717 & 76.3 & 15489 & 72.1 & 7317 & 76.7 & 5704 & 72.2 \\ \text { One } & 2815 & 12.8 & 3084 & 14.3 & 1161 & 12.2 & 1054 & 13.3 \\ \text { Two } & 1034 & 4.7 & 1246 & 5.8 & 458 & 4.8 & 467 & 5.9 \\ 3-5 & 779 & 3.6 & 973 & 4.5 & 348 & 3.6 & 390 & 4.9 \\ \text { 6 or more } & 577 & 2.6 & 703 & 3.2 & 253 & 2.7 & 284 & 3.6 \\ \text { TOTAL } & 21922 & 100.0 & 21495 & 100.0 & 9537 & 100.0 & 7899 & 100.0\end{array}$

Number of health problems (Q13)

\begin{tabular}{lcccccccc} 
None & 1959 & 8.9 & 1237 & 5.8 & 944 & 9.9 & 528 & 6.7 \\
One & 4088 & 18.6 & 3466 & 16.1 & 1995 & 20.9 & 1485 & 18.8 \\
Two & 6018 & 27.5 & 5781 & 26.9 & 2597 & 27.2 & 2194 & 27.8 \\
Three & 4537 & 20.7 & 4985 & 23.2 & 1814 & 19.0 & 1684 & 21.3 \\
4 or more & 5320 & 24.3 & 6026 & 28.0 & 2187 & 22.9 & 2008 & 25.4 \\
TOTAL & 21922 & 100.0 & 21495 & 100.0 & 9537 & 100.0 & 7899 & 100.0 \\
\hline
\end{tabular}

the very oldest. Very few women were employed at the census, with the most common job coded as a day labourer in all age groups. In men the proportion not working increased with age at census, with about a third of the oldest group of men (>75 years in 2005) being classified as 'dependent'. Farming was by far the most common occupation among men of all ages. Classification by socioeconomic group depended on the assets of the household, with few coded as 'very poor or destitute'. 
Table 2 Distributions of education, occupation, socioeconomic group and smoking by age (from census year of birth) and sex: census-matched subpopulation $(\mathrm{N}=17436)$

\begin{tabular}{|c|c|c|c|c|c|c|c|c|c|c|c|c|}
\hline \multicolumn{5}{|c|}{ Men } & \multicolumn{8}{|c|}{ Women } \\
\hline \multicolumn{5}{|c|}{ Age (years) } & \multicolumn{8}{|c|}{ Age (years) } \\
\hline $65-69$ & $70-74$ & $75-79$ & $>80$ & Total & $65-69$ & & & & & & & Total \\
\hline$\%$ & $\%$ & $\%$ & $\mathrm{~N}$ & $\%$ & $\%$ & $\mathrm{~N}$ & $\%$ & $\mathrm{~N}$ & $\%$ & $\mathbf{N}$ & $\%$ & $\mathbf{N}$ \\
\hline
\end{tabular}

\section{Education}

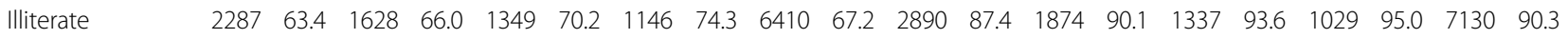

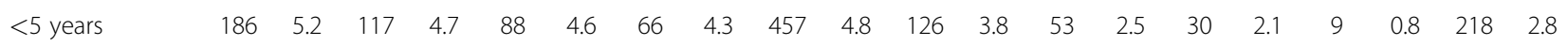

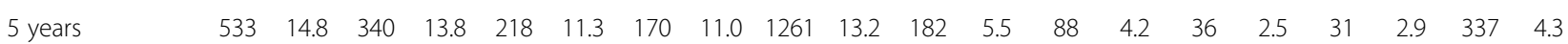

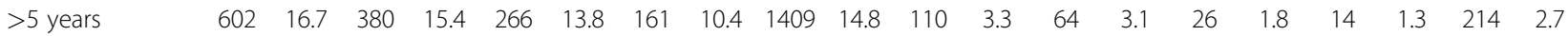

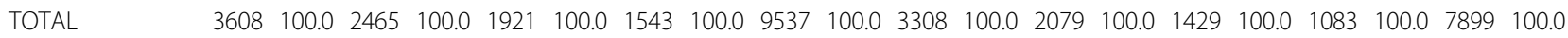

\section{Occupation at census}

$\begin{array}{lccccccccccccccccccccccc}\begin{array}{l}\text { Dependent/ } \\ \text { housewife/UE }\end{array} & 366 & 10.1 & 324 & 13.1 & 459 & 23.9 & 532 & 34.5 & 1681 & 17.6 & 3221 & 97.4 & 2032 & 97.7 & 1399 & 97.9 & 1060 & 97.9 & 7712 & 97.6 \\ \text { Farmer } & 1971 & 54.6 & 1394 & 56.6 & 96 & 50.3 & 710 & 46.0 & 5041 & 52.9 & 15 & 0.5 & 8 & 0.4 & 3 & 0.2 & 3 & 0.3 & 29 & 0.4 \\ \text { Business } & 535 & 14.8 & 343 & 13.9 & 230 & 12.0 & 134 & 8.7 & 1242 & 13.0 & 12 & 0.4 & 9 & 0.4 & 8 & 0.6 & 4 & 0.4 & 33 & 0.4 \\ \text { Day labourer } & 280 & 7.8 & 171 & 6.9 & 103 & 5.4 & 76 & 4.9 & 630 & 6.6 & 35 & 1.1 & 17 & 0.8 & 10 & 0.7 & 6 & 0.6 & 68 & 0.9 \\ \text { Service } & 209 & 5.8 & 104 & 4.2 & 72 & 3.7 & 45 & 2.9 & 430 & 4.5 & 13 & 0.4 & 2 & 0.1 & 3 & 0.2 & 4 & 0.4 & 22 & 0.3 \\ \text { Craftsman } & 66 & 1.8 & 36 & 1.5 & 22 & 1.1 & 13 & 0.8 & 137 & 1.4 & 0 & 0.0 & 2 & 0.1 & 0 & 0.0 & 0 & 0.0 & 2 & 0.0 \\ \text { Other } & 181 & 5.0 & 93 & 3.8 & 69 & 3.6 & 33 & 2.1 & 376 & 3.9 & 12 & 0.4 & 9 & 0.4 & 6 & 0.4 & 6 & 0.6 & 33 & 0.4\end{array}$

TOTAL $\quad \begin{array}{llllllllllllllllllll}3608 & 100.0 & 2465 & 100.0 & 1921 & 100.0 & 1543 & 100.0 & 9537 & 100.0 & 3308 & 100.0 & 2079 & 100.0 & 1429 & 100.0 & 1083 & 100.0 & 7899 & 100.0\end{array}$

\section{Socioeconomics group (of household)}

$\begin{array}{lcccccccccccccccccccc}\text { Destitute (Aw) } & 44 & 1.2 & 32 & 1.3 & 20 & 1.0 & 23 & 1.5 & 119 & 1.2 & 78 & 2.4 & 66 & 3.2 & 42 & 2.9 & 29 & 2.7 & 215 & 2.7 \\ \text { Very poor (Ah) } & 6 & 0.2 & 2 & 0.1 & 7 & 0.4 & 3 & 0.2 & 18 & 0.2 & 30 & 0.9 & 16 & 0.8 & 20 & 1.4 & 20 & 1.8 & 86 & 1.1 \\ \text { Poor (Ka) } & 2201 & 61.0 & 1439 & 58.4 & 1112 & 59.9 & 828 & 53.7 & 5580 & 58.5 & 2035 & 61.5 & 1237 & 59.5 & 866 & 60.6 & 625 & 57.3 & 4763 & 60.3 \\ \text { Middle class (Kha) } & 1119 & 31.0 & 811 & 32.9 & 629 & 32.7 & 538 & 34.9 & 3097 & 32.5 & 983 & 29.7 & 613 & 29.5 & 409 & 28.6 & 323 & 29.8 & 2328 & 29.5 \\ \text { Wealthier (Ga) } & 238 & 6.6 & 181 & 7.3 & 153 & 8.0 & 151 & 9.8 & 723 & 7.6 & 182 & 5.5 & 147 & 7.1 & 92 & 6.4 & 86 & 7.9 & 507 & 6.4 \\ \text { TOTAL } & 3608 & 100.0 & 2465 & 100.0 & 1921 & 100.0 & 1543 & 100.0 & 9537 & 100.0 & 3308 & 100.0 & 2079 & 100.0 & 1429 & 100.0 & 1083 & 100.0 & 7899 & 100.0\end{array}$

Smoker

No

Yes

TOTAL

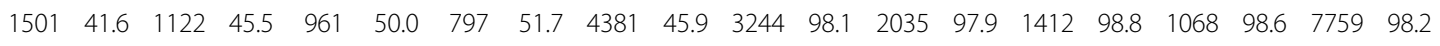

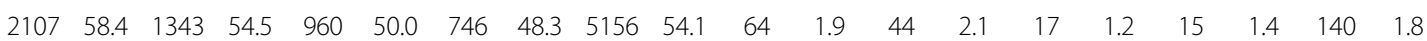

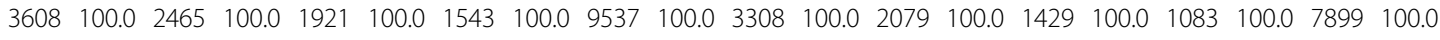

About half the men but few women were smokers at the time of the census.

\section{Disability}

Reporting of difficulty (code 3 or 4) increased steadily with age for all disabilities, with women more likely than men to report disability at almost every age (Table 3). The ranking of disabilities was very similar at each age, with difficulties in lifting and carrying, seeing, and going for some distance outside the home being rated as 'much difficulty' or 'can't do at all' by some $20 \%$ to $50 \%$ of those aged 85 or greater. The final column in Table 3 shows the number for whom the paramedic reported the difficulty underestimated. These were uniformly low.
The reporting of troublesome heath conditions followed a similar pattern to that for disability (Table 4) with only uterine prolapse and sexual difficulties not showing increase with age, in women. Painful joints were by far the most common symptom, with little increase in the proportion reporting this symptom once the age of 70 years had been reached. In those $\geq 85$ years chest pain and urinary incontinence were, for both men and women, the second and third most common condition. The recoding of 'other' problems was not related to age. Responses to this question had been coded to capture reports of diabetes, hypertension, digestive system problems and back pain, but the number reporting each condition was small.

Results of 12 logistic regression analyses relating specific disability to socio-demographic and heath conditions 
Table 3 Number $(n)$ reporting 'much difficulty 'or 'can't do it at all' for each functional capacity (Q 1-12) by age and $\operatorname{sex}(N=43112)$

\begin{tabular}{|c|c|c|c|c|c|c|c|c|c|c|c|c|c|c|c|c|c|c|}
\hline & \multicolumn{2}{|c|}{$<60$} & \multicolumn{2}{|c|}{$60<65$} & \multicolumn{2}{|c|}{$65<70$} & \multicolumn{2}{|c|}{$70<75$} & \multicolumn{2}{|c|}{$75<80$} & \multicolumn{2}{|c|}{$80<85$} & \multicolumn{2}{|c|}{$>85$} & \multicolumn{2}{|c|}{ TOTAL } & \multirow[t]{2}{*}{$\%$ under-ratec } \\
\hline & & $n$ & $\%$ & $\mathrm{n}$ & $\%$ & $\mathrm{n}$ & $\%$ & $\mathrm{n}$ & $\%$ & $n$ & $\%$ & $\mathrm{n}$ & $\%$ & $\mathrm{n}$ & $\%$ & $n$ & $\%$ & \\
\hline \multirow[t]{2}{*}{ Q1 seeing } & men & 13 & 0.9 & 116 & 3.0 & 217 & 4.3 & 316 & 6.3 & 262 & 9.7 & 252 & 12.7 & 362 & 20.0 & 1538 & 7.1 & 1.6 \\
\hline & women & 34 & 2.1 & 218 & 4.3 & 285 & 5.1 & 413 & 9.8 & 211 & 12.6 & 302 & 18.8 & 379 & 26.1 & 1842 & 8.6 & 2.1 \\
\hline \multirow[t]{2}{*}{ Q2 hearing } & men & 2 & 0.1 & 61 & 1.6 & 75 & 1.5 & 142 & 2.8 & 116 & 4.3 & 151 & 7.6 & 204 & 11.3 & 751 & 3.4 & 0.8 \\
\hline & women & 10 & 0.6 & 96 & 1.9 & 135 & 2.4 & 213 & 5.1 & 91 & 5.4 & 139 & 8.6 & 230 & 15.8 & 914 & 4.3 & 1.1 \\
\hline \multirow[t]{2}{*}{ Q3 getting up } & men & 8 & 0.6 & 47 & 1.2 & 67 & 1.3 & 93 & 1.8 & 81 & 3.0 & 99 & 5.0 & 206 & 11.4 & 601 & 2.8 & 0.2 \\
\hline & women & 7 & 0.4 & 60 & 1.2 & 74 & 1.3 & 130 & 3.1 & 72 & 4.3 & 140 & 8.7 & 228 & 15.7 & 711 & 3.3 & 0.3 \\
\hline \multirow[t]{2}{*}{ Q4 standing } & men & 9 & 0.7 & 36 & 0.9 & 75 & 1.5 & 101 & 2.0 & 94 & 3.5 & 118 & 5.9 & 223 & 12.3 & 656 & 3.0 & 0.3 \\
\hline & women & 6 & 0.4 & 43 & 0.8 & 68 & 1.2 & 137 & 3.3 & 79 & 4.7 & 161 & 10.0 & 283 & 19.5 & 777 & 3.6 & 0.2 \\
\hline \multirow[t]{2}{*}{ Q5 walking } & men & 4 & 0.3 & 34 & 0.9 & 63 & 1.2 & 87 & 1.7 & 75 & 2.8 & 93 & 4.7 & 189 & 10.4 & 545 & 2.5 & 0.2 \\
\hline & women & 7 & 0.4 & 42 & 0.8 & 57 & 1.0 & 119 & 2.8 & 68 & 4.1 & 141 & 8.8 & 227 & 15.6 & 661 & 3.1 & 0.2 \\
\hline \multirow[t]{2}{*}{ Q6 go outside } & men & 9 & 0.7 & 74 & 1.9 & 142 & 2.8 & 201 & 4.0 & 169 & 6.2 & 202 & 10.2 & 354 & 19.6 & 115 & 5.3 & 0.3 \\
\hline & women & 12 & 0.7 & 101 & 2.0 & 191 & 3.4 & 303 & 7.2 & 174 & 10.4 & 293 & 18.2 & 426 & 29.3 & 1500 & 7.0 & 0.3 \\
\hline \multirow[t]{2}{*}{ Q7 washing } & men & 2 & 0.1 & 37 & 1.0 & 70 & 1.4 & 92 & 1.8 & 74 & 2.7 & 114 & 5.7 & 213 & 11.8 & 602 & 2.8 & 0.1 \\
\hline & women & 10 & 0.6 & 38 & 0.7 & 72 & 1.3 & 127 & 3.0 & 79 & 4.7 & 152 & 9.4 & 253 & 17.4 & 731 & 3.4 & 0.2 \\
\hline \multirow[t]{2}{*}{ Q8 lavatory } & men & 4 & 0.3 & 35 & 0.9 & 70 & 1.4 & 84 & 1.7 & 68 & 2.5 & 92 & 4.6 & 196 & 10.8 & 549 & 2.5 & 0.1 \\
\hline & women & 6 & 0.4 & 41 & 0.8 & 63 & 1.1 & 122 & 2.9 & 77 & 4.6 & 139 & 8.6 & 228 & 15.7 & 676 & 3.2 & 0.1 \\
\hline \multirow[t]{2}{*}{ Q9 understanding } & men & 11 & 0.8 & 65 & 1.7 & 123 & 2.4 & 153 & 3.0 & 127 & 4.7 & 161 & 8.1 & 259 & 14.3 & 899 & 4.1 & 0.4 \\
\hline & women & 10 & 0.6 & 117 & 2.3 & 201 & 3.6 & 230 & 5.5 & 124 & 7.4 & 169 & 10.5 & 283 & 19.5 & 1134 & 5.3 & 0.5 \\
\hline \multirow[t]{2}{*}{ Q10 remembering } & men & 4 & 0.3 & 43 & 1.1 & 92 & 1.8 & 110 & 2.2 & 95 & 3.5 & 112 & 5.6 & 179 & 9.9 & 635 & 2.9 & 0.6 \\
\hline & women & 12 & 0.7 & 78 & 1.5 & 119 & 2.1 & 158 & 3.7 & 84 & 5.0 & 129 & 8.0 & 228 & 15.7 & 808 & 3.8 & 0.6 \\
\hline \multirow[t]{2}{*}{ Q11 lifting/carrying } & men & 51 & 3.7 & 487 & 12.7 & 699 & 13.9 & 792 & 15.7 & 609 & 22.5 & 540 & 27.2 & 672 & 37.2 & 3850 & 17.7 & 1.5 \\
\hline & women & 105 & 6.4 & 700 & 13.7 & 857 & 15.2 & 991 & 23.5 & 483 & 28.9 & 592 & 36.8 & 702 & 48.7 & 4430 & 20.8 & 1.7 \\
\hline \multirow[t]{2}{*}{ Q12 getting food } & men & 22 & 1.6 & 81 & 2.1 & 83 & 1.6 & 108 & 2.1 & 75 & 2.8 & 73 & 3.7 & 87 & 4.8 & 529 & 2.4 & 1.3 \\
\hline & women & 36 & 2.2 & 101 & 2.0 & 115 & 2.0 & 122 & 2.9 & 66 & 3.9 & 84 & 5.2 & 118 & 8.1 & 642 & 3.0 & 1.6 \\
\hline \multirow[t]{2}{*}{ TOTAL N } & men & 1369 & & 3836 & & 5043 & & 5033 & & 2705 & & 1988 & & 1807 & & 21781 & & - \\
\hline & women & 1653 & & 5102 & & 5628 & & 4215 & & 1672 & & 1608 & & 1453 & & 21331 & & - \\
\hline
\end{tabular}

showed each disability remained significantly related to increasing age in the full model (except for 'getting enough to eat 'for men) (Tables 5 and 6). Widowhood (no living spouse) was associated with the reporting of all but one disability for women and for 9/12 disabilities for men. In contrast, the small number of 'very poor or destitute' were not at greater risk (compared to the wealthiest in these villages), except for not getting enough to eat. Illiteracy was most strongly related, for both men and women, with difficulties seeing and hearing, going outside and lifting and carrying heavy loads. A man not working at the census was more likely to be disabled at the time of the survey (having adjusted for age), being more at risk on 7 of the 12 functional capacities. Smoking was not related to disability.

The relation between disability and troublesome health conditions varied markedly with the type of ill-health.
Painful joints, although the most common complaint, were related only to difficulty lifting whereas hemiplegia and resting tremor were associated with increased risk of reporting every dimension of disability. In men, but not in women, breathing problems were commonly associated with disability. Chest pain showed little consistent relation to disability. Further analysis indicated a strong relation between depression and reports of chest pain: $55.9 \%$ (5345/9556) of those saying that depression often made life difficult reported chest pain compared to $39.3 \%(13307 / 33861)$ of those not reporting depression. Inclusion of depression in the model attenuated the bivariate relation between chest pain and, for example, difficulty walking in the home. Overall, the relation between depression and reported disabilities was striking (Tables 5 and 6): a subject who was 'very often depressed' was more likely to report difficulties on each 
Table 4 Number $(n)$ reporting each health problem $(Q 13-14)$ by age (years) and sex $(N=43,112)$

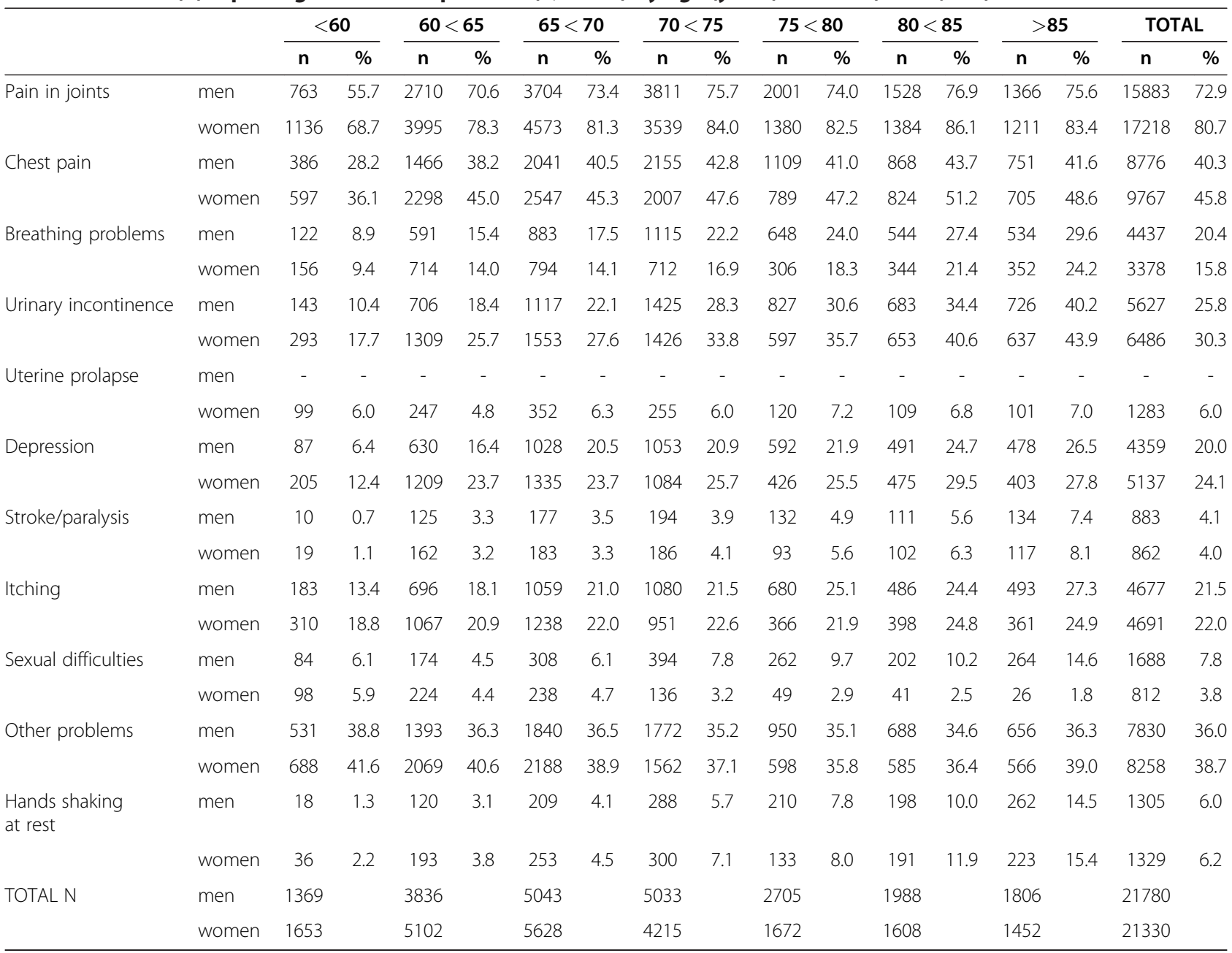

of the disability questions (except hearing for women). The relation of disability to urinary incontinence was at least as strong, with both men and women who reported troublesome urinary incontinence being more likely to report each disability (except, for women, not getting enough food). In all age groups there was a close relation between urinary incontinence and depression, with depression reported overall in $41.0 \%$ of those who reported troublesome urinary incontinence but only $14.6 \%$ of those who did not. In a linear regression in which total disability score was the dependent variable, urinary incontinence was more strongly related to overall disability than any health problem except hemiplegia tremor and resting tremor (Table 7).

\section{Help received and needed}

More than half the oldest group (over 85 years) had some help from a family member (Table 8): help from someone outside the family was mentioned by only 39 : of 11211 villagers with 'much difficult' on at least one functional capacity, only 15 reported getting help outside the family. For women help was most commonly from a daughter-in-law, with mobility and bathing the most common assistance. In a logistic regression analysis, with any help reported (or not) as the outcome, help received was reported somewhat more frequently by women, by those with no living spouse, those who were older and those with a higher disability score (Table 9).

Nearly two thirds of both men and women in the survey reported that they were in need of help. The help specified was most usually treatment for a medical condition or financial aid (Table 8).

\section{Discussion}

This survey of disability in some 43,000 villagers believed to be aged $\geq 60$ years found that only a minority (26\%) reported 'much difficulty' on any of 12 functional capacities. The proportion increased markedly with age and amongst the most elderly ( $\geq 85$ years) there were widespread problems, in lifting and carrying, with 
Table 5 Relation of disabilities to social factors and illness (Functional capacities 1-6): multivariate logistic regression $(\mathrm{N}=17436)$

\begin{tabular}{|c|c|c|c|c|c|c|c|c|c|c|c|c|c|}
\hline \multirow[t]{3}{*}{ 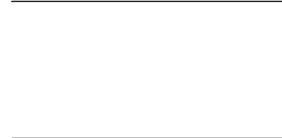 } & \multirow[t]{3}{*}{ Sex } & \multicolumn{12}{|c|}{ Disability } \\
\hline & & \multicolumn{2}{|c|}{ Q1 seeing } & \multicolumn{2}{|c|}{ Q2 hearing } & \multicolumn{2}{|c|}{ Q3 getting up } & \multicolumn{2}{|c|}{ Q4 standing } & \multicolumn{2}{|c|}{ Q5 walking } & \multicolumn{2}{|c|}{ Q6 go outside } \\
\hline & & OR & $95 \% \mathrm{Cl}$ & OR & $95 \% \mathrm{Cl}$ & OR & $95 \% \mathrm{Cl}$ & OR & $95 \% \mathrm{Cl}$ & OR & $95 \% \mathrm{Cl}$ & OR & $95 \% \mathrm{Cl}$ \\
\hline \multicolumn{14}{|l|}{ Sociodemographic } \\
\hline \multirow{2}{*}{$\begin{array}{l}\text { Age from census } \\
\text { (continuous variable) }\end{array}$} & men & 1.04 & $1.03-1.05$ & 1.04 & $1.02-1.05$ & 1.06 & $1.04-1.07$ & 1.06 & $1.05-1.08$ & 1.05 & $1.03-1.07$ & 1.05 & $1.04-1.63$ \\
\hline & women & 1.03 & $1.02-1.04$ & 1.04 & $1.03-1.73$ & 1.04 & 1.03-1.06 & 1.05 & $1.03-1.06$ & 1.04 & $1.02-1.06$ & 1.04 & $1.03-1.06$ \\
\hline \multirow[t]{2}{*}{ No living spouse } & men & 1.37 & $1.14-1.64$ & 1.28 & $1.02-1.61$ & 1.21 & $0.91-1.60$ & 1.21 & $0.93-1.57$ & 1.21 & $0.90-1.62$ & 1.51 & $1.24-1.85$ \\
\hline & women & 1.42 & $1.23-1.65$ & 1.45 & $1.21-1.73$ & 1.51 & $1.24-1.85$ & 1.61 & $1.32-1.98$ & 1.48 & $1.21-1.82$ & 1.68 & $1.41-2.00$ \\
\hline \multirow[t]{2}{*}{ Very poor/destitute* } & men & 1.20 & $0.65-2.22$ & 1.84 & $0.82-4.11$ & 0.37 & $0.09-1.54$ & 0.64 & $0.21-1.95$ & 0.39 & $0.09-1.65$ & 0.42 & $0.16-1.93$ \\
\hline & women & 1.34 & $0.83-2.18$ & 1.00 & $0.50-2.00$ & 1.29 & $0.58-2.85$ & 1.17 & $0.54-2.52$ & 1.02 & $0.43-2.42$ & 0.81 & $0.46-1.43$ \\
\hline \multirow[t]{2}{*}{ Illiterate } & men & 1.34 & $1.11-1.62$ & 1.31 & $1.01-1.72$ & 1.10 & $0.80-1.49$ & 1.31 & $0.85-1.53$ & 1.18 & $0.85-1.63$ & 1.23 & $0.99-1.54$ \\
\hline & women & 1.70 & $1.20-2.40$ & 1.42 & $0.89-2.29$ & 0.97 & $0.60-1.57$ & 1.45 & $0.86-2.48$ & 1.27 & $0.74-2.16$ & 1.75 & $1.18-2.61$ \\
\hline \multirow[t]{2}{*}{ No job at census } & men & 1.33 & $1.10-1.61$ & 1.62 & $1.24-2.10$ & 1.29 & $0.94-1.77$ & 1.31 & $0.97-1.77$ & 1.46 & $1.06-2.01$ & 1.35 & $1.08-1.70$ \\
\hline & women & 1.29 & $0.74-2.22$ & 0.83 & $0.42-1.64$ & 1.38 & $0.52-3.32$ & 1.45 & $0.57-3.65$ & 1.13 & $0.45-2.55$ & 1.61 & $0.80-3.22$ \\
\hline \multirow[t]{2}{*}{ Smoker at census } & men & 0.88 & $0.75-1.03$ & 1.00 & $0.80-1.25$ & 0.84 & $0.64-1.10$ & 0.95 & $0.73-1.23$ & 0.86 & $0.65-1.14$ & 1.03 & $0.85-1.25$ \\
\hline & women & 0.50 & $0.23-1.09$ & 0.49 & $0.15-1.56$ & 0.61 & $0.18-2.07$ & 0.57 & $0.17-1.96$ & 0.67 & $0.20-2.31$ & 0.70 & $0.31-1.57$ \\
\hline \multicolumn{14}{|l|}{ Disease/condition } \\
\hline \multirow[t]{2}{*}{ Pain in joints } & men & 1.13 & $0.93-1.37$ & 1.17 & $0.89-1.55$ & 1.00 & $0.73-1.37$ & 1.06 & $0.79-1.44$ & 1.21 & $0.87-1.70$ & 1.20 & $0.95-1.51$ \\
\hline & women & 1.30 & $1.04-1.63$ & 1.02 & $0.75-1.38$ & 1.05 & $0.75-1.47$ & 0.84 & $0.61-1.15$ & 0.81 & $0.58-1.13$ & 1.26 & $0.99-1.61$ \\
\hline \multirow[t]{2}{*}{ Chest pain } & men & 1.25 & $1.05-1.47$ & 1.46 & $1.15-1.85$ & 1.00 & $0.75-1.33$ & 0.86 & $0.65-1.13$ & 0.88 & $0.66-1.19$ & 0.97 & $0.90-1.19$ \\
\hline & women & 1.28 & $1.08-1.51$ & 1.31 & $1.03-1.66$ & 0.64 & $0.48-0.85$ & 0.68 & $0.52-0.89$ & 0.73 & $0.54-0.97$ & 0.85 & $0.71-1.03$ \\
\hline \multirow[t]{2}{*}{ Breathing problem } & men & 1.48 & $1.24-1.77$ & 1.31 & $1.02-1.68$ & 1.50 & $1.12-2.01$ & 1.73 & $1.32-2.28$ & 1.46 & $1.08-1.97$ & 1.45 & $1.18-1.79$ \\
\hline & women & 1.30 & $1.06-1.58$ & 1.15 & $0.87-1.51$ & 1.32 & $0.96-1.81$ & 1.29 & $0.95-1.75$ & 1.26 & $0.91-1.74$ & 1.23 & $0.98-1.53$ \\
\hline \multirow[t]{2}{*}{ Incontinence } & men & 1.52 & $1.28-1.81$ & 1.69 & $1.32-2.15$ & 1.61 & $1.21-2.15$ & 1.58 & $1.20-2.08$ & 1.58 & $1.17-2.12$ & 1.87 & $1.52-2.29$ \\
\hline & women & 1.41 & $1.19-1.67$ & 1.54 & $1.21-1.95$ & 1.81 & $1.37-2.39$ & 2.06 & $1.58-2.69$ & 1.83 & $1.38-2.44$ & 1.80 & $1.49-2.18$ \\
\hline \multirow[t]{2}{*}{ Depression } & men & 1.56 & $1.30-1.88$ & 1.32 & $1.02-1.72$ & 1.60 & $1.18-2.16$ & 1.46 & $1.09-1.95$ & 1.69 & $1.24-2.29$ & 1.63 & $1.31-2.01$ \\
\hline & women & 1.53 & $1.28-1.83$ & 1.26 & $0.98-1.62$ & 1.38 & $1.03-1.85$ & 1.42 & $1.08-1.87$ & 1.29 & $0.96-1.74$ & 1.48 & $1.21-1.80$ \\
\hline \multirow[t]{2}{*}{ Paralysis } & men & 1.09 & $0.78-1.53$ & 1.33 & $0.87-2.04$ & 9.06 & $6.61-12.41$ & 8.53 & $6.28-11.58$ & 10.20 & $7.42-14.02$ & 4.90 & $3.75-6.41$ \\
\hline & women & 1.79 & $1.33-2.41$ & 0.98 & $0.62-1.54$ & 13.68 & $10.10-18.53$ & 11.56 & $8.56-15.60$ & 13.67 & $10.04-18.61$ & 6.10 & $4.68-7.95$ \\
\hline \multirow[t]{2}{*}{ Itching } & men & 0.99 & $0.83-1.19$ & 0.87 & $0.67-1.14$ & 0.77 & $0.56-1.06$ & 0.75 & $0.55-1.01$ & 0.71 & $0.51-0.99$ & 0.91 & $0.73-1.13$ \\
\hline & women & 0.91 & $0.75-1.10$ & 1.33 & $1.04-1.70$ & 0.78 & $0.57-1.07$ & 0.92 & $0.68-1.24$ & 0.88 & $0.64-1.22$ & 0.96 & $0.78-1.19$ \\
\hline \multirow[t]{2}{*}{ Other } & men & 1.57 & $1.34-1.84$ & 1.37 & $1.09-1.72$ & 1.38 & $1.05-1.82$ & 1.44 & $1.11-1.86$ & 1.46 & $1.11-1.94$ & 1.42 & $1.17-1.72$ \\
\hline & women & 1.62 & $1.38-1.90$ & 1.52 & $1.22-1.89$ & 1.53 & $1.18-1.98$ & 1.59 & $1.24-2.04$ & 1.50 & $1.15-1.96$ & 1.51 & $1.26-1.80$ \\
\hline Shaking at rest & men & 1.97 & $1.53-2.53$ & 2.59 & $1.90-3.55$ & 3.92 & $2.84-5.41$ & 3.89 & $2.85-5.30$ & 4.4 & $3.21-6.14$ & 2.89 & $2.23-3.74$ \\
\hline & women & 2.58 & $2.05-3.25$ & 3.17 & $2.36-4.25$ & 3.14 & $2.26-4.35$ & 3.57 & $2.62-4.86$ & 3.51 & $2.53-4.88$ & 2.53 & $1.97-3.25$ \\
\hline
\end{tabular}

- Relative to wealthier.

eyesight and with going outside the house for any distance. It is of note that only $29 \%$ of the elderly villagers reported receiving any help from their family members and virtually none had help from outside the family. However those receiving help from the family did appear to be those with the greatest needs.

The study was set up to find ways in which the extent and impact of disabilities could be lessened by appropriate interventions. The high disability rate among those with hemiplegia was expected but the recent introduction by GK of community physiotherapists may help to ensure that a greater proportion of survivors have rapid and appropriate rehabilitation. The comprehensive range of disability among those with a resting tremor is also of interest and would warrant a more focused inquiry: those reporting the symptom here are unlikely to have been 
Table 6 Relation of disabilities to social factors and illness (Functional capacities 7-12): multivariate logistic regression ( $N=17436)$

\begin{tabular}{|c|c|c|c|c|c|c|c|c|c|c|c|c|c|}
\hline & \multirow[t]{3}{*}{ Sex } & \multicolumn{12}{|c|}{ Disability } \\
\hline & & \multicolumn{2}{|c|}{ Q7 bath } & \multicolumn{2}{|c|}{ Q8 lavatory } & \multicolumn{2}{|c|}{ Q9 understand } & \multicolumn{2}{|c|}{ Q10 Memory } & \multicolumn{2}{|c|}{ Q11 lifting } & \multicolumn{2}{|c|}{ Q12 food } \\
\hline & & OR & $95 \% \mathrm{Cl}$ & OR & $95 \% \mathrm{Cl}$ & OR & $95 \% \mathrm{Cl}$ & OR & $95 \% \mathrm{Cl}$ & OR & $95 \% \mathrm{Cl}$ & OR & $95 \% \mathrm{Cl}$ \\
\hline \multicolumn{14}{|l|}{ Sociodemographic } \\
\hline \multirow[t]{2}{*}{ Age from census } & men & 1.05 & $1.03-1.06$ & 1.05 & $1.03-1.07$ & 1.04 & $1.03-1.06$ & 1.04 & $1.03-1.06$ & 1.02 & $1.02-1.03$ & 1.01 & $0.99-1.06$ \\
\hline & women & 1.04 & $1.03-1.06$ & 1.05 & $1.03-1.07$ & 1.04 & $1.03-1.05$ & 1.05 & $1.03-1.06$ & 1.03 & $1.02-1.04$ & 1.03 & $1.01-1.05$ \\
\hline \multirow[t]{2}{*}{ No living spouse } & men & 1.42 & $1.13-1.80$ & 1.36 & $1.06-1.74$ & 1.50 & $1.22-1.84$ & 1.51 & $1.20-1.89$ & 1.41 & $1.21-1.63$ & 1.38 & $1.04-1.84$ \\
\hline & women & 1.47 & $1.21-1.80$ & 1.55 & $1.27-1.91$ & 1.37 & $0.55-2.04$ & 1.39 & $1.15-1.68$ & 1.47 & $1.31-1.66$ & 1.29 & $1.02-1.62$ \\
\hline \multirow[t]{2}{*}{ Very poor/destitute } & men & 0.55 & $0.16-1.93$ & 0.39 & $0.10-1.59$ & 0.89 & $0.37-2.14$ & 0.56 & $0.20-1.61$ & 0.55 & $0.32-0.89$ & 3.86 & $1.47-10.16$ \\
\hline & women & 1.45 & $0.67-3.13$ & 1.12 & $0.50-2.49$ & 1.06 & $0.55-2.04$ & 0.82 & $0.42-1.62$ & 0.71 & $0.50-1.03$ & 5.14 & 2.33-11.35 \\
\hline \multirow[t]{2}{*}{ Illiterate } & men & 1.08 & $0.80-1.47$ & 1.00 & $0.73-1.36$ & 1.04 & $0.82-1.31$ & 1.21 & $0.91-1.62$ & 1.35 & $1.19-1.54$ & 1.03 & $0.71-1.49$ \\
\hline & women & 1.19 & 0.73-1.95 & 1.35 & $0.78-2.33$ & 1.96 & $1.24-3.10$ & 1.91 & $1.11-3.30$ & 1.53 & $1.22-1.91$ & 1.39 & $0.74-2.63$ \\
\hline \multirow[t]{2}{*}{ No job at census } & men & 1.53 & $1.12-2.07$ & 1.53 & $1.11-2.11$ & 1.48 & $1.16-1.89$ & 1.07 & $0.79-1.45$ & 1.14 & $0.98-1.32$ & 1.10 & $0.73-1.64$ \\
\hline & women & 1.00 & $0.45-2.22$ & 1.17 & $0.47-2.93$ & 0.78 & $0.43-1.43$ & 0.62 & $0.33-1.19$ & 1.18 & $0.79-1.77$ & 0.51 & $0.28-0.93$ \\
\hline \multirow[t]{2}{*}{ Smoker at census } & men & 1.08 & $0.83-1.41$ & 1.06 & $0.80-1.41$ & 1.07 & $0.87-1.32$ & 0.83 & $0.64-1.06$ & 1.11 & $0.99-1.24$ & 0.91 & $0.66-1.26$ \\
\hline & women & 1.57 & $0.69-3.58$ & 0.71 & $0.21-2.39$ & 0.79 & $0.34-1.82$ & 0.78 & $0.29-2.18$ & 0.60 & $0.35-1.00$ & 0.91 & $0.32-2.29$ \\
\hline \multicolumn{14}{|l|}{ Disease/condition } \\
\hline \multirow[t]{2}{*}{ Pain in joints } & men & 1.05 & $0.77-1.44$ & 1.21 & $0.80-1.41$ & 1.15 & $0.90-1.49$ & 1.08 & $0.80-1.45$ & 1.15 & $1.01-1.32$ & 1.25 & $0.82-1.90$ \\
\hline & women & 0.90 & $0.65-1.23$ & 1.05 & $0.74-1.49$ & 1.15 & $0.88-1.51$ & 0.88 & $0.64-1.20$ & 1.31 & $1.12-1.53$ & 0.90 & $0.61-1.33$ \\
\hline \multirow[t]{2}{*}{ Chest pain } & men & 0.79 & $0.60-1.05$ & 0.82 & $0.61-1.11$ & 1.26 & $1.02-1.56$ & 0.98 & $0.75-1.27$ & 1.11 & $0.98-1.23$ & 1.26 & $0.90-1.76$ \\
\hline & women & 0.72 & $0.55-0.93$ & 0.68 & $0.51-0.91$ & 1.10 & $0.89-1.35$ & 0.99 & $0.77-1.28$ & 1.06 & $0.94-1.70$ & 1.17 & $0.86-1.60$ \\
\hline \multirow[t]{2}{*}{ Breathing problem } & men & 1.53 & $1.16-2.03$ & 1.36 & $1.01-1.84$ & 1.06 & $0.84-1.34$ & 1.22 & $0.93-1.62$ & 1.30 & $1.14-1.49$ & 1.92 & $1.37-2.67$ \\
\hline & women & 1.24 & $0.91-1.69$ & 0.99 & $0.70-1.39$ & 1.25 & $0.98-1.60$ & 0.82 & $0.60-1.13$ & 1.15 & $0.98-1.34$ & 1.66 & $1.19-2.31$ \\
\hline \multirow[t]{2}{*}{ Incontinence } & men & 1.66 & $1.26-2.20$ & 1.75 & $1.31-2.35$ & 1.55 & $1.24-1.93$ & 1.55 & $1.19-2.03$ & 1.71 & $1.51-1.94$ & 1.99 & $1.42-2.78$ \\
\hline & women & 1.63 & $1.25-2.14$ & 1.66 & $1.25-2.21$ & 1.30 & $1.05-1.61$ & 1.75 & $1.36-2.24$ & 1.37 & $1.21-1.56$ & 1.11 & $0.81-1.52$ \\
\hline \multirow[t]{2}{*}{ Depression } & men & 1.72 & $1.29-2.29$ & 1.70 & $1.26-2.31$ & 1.59 & $1.26-2.01$ & 1.83 & $1.39-2.42$ & 1.61 & $1.40-1.84$ & 1.87 & $1.32-2.64$ \\
\hline & women & 1.37 & $1.03-1.82$ & 1.44 & $1.07-1.93$ & 1.17 & $0.93-1.47$ & 1.58 & $1.22-2.05$ & 1.39 & $1.21-1.59$ & 2.19 & $1.60-3.00$ \\
\hline \multirow[t]{2}{*}{ Paralysis } & men & 7.97 & $5.83-10.89$ & 8.42 & $6.11-11.62$ & 1.74 & $1.22-2.49$ & 2.56 & $1.76-3.72$ & 2.47 & $1.97-3.09$ & 2.06 & $1.26-3.36$ \\
\hline & women & 11.64 & $8.61-15.72$ & 12.23 & $8.94-16.74$ & 2.12 & $1.51-2.97$ & 3.18 & $2.23-4.52$ & 3.30 & $2.60-4.19$ & 3.08 & $2.01-4.72$ \\
\hline \multirow[t]{2}{*}{ Itching } & men & 0.84 & $0.62-1.14$ & 0.75 & $0.55-1.04$ & 1.38 & $1.11-1.73$ & 1.00 & $0.76-1.33$ & 1.04 & $0.91-1.19$ & 1.14 & $0.81-1.61$ \\
\hline & women & 0.85 & $0.63-1.15$ & 0.93 & $0.67-1.27$ & 1.41 & $1.51-2.97$ & 1.13 & $0.93-1.54$ & 1.10 & $0.96-1.27$ & 1.45 & $1.06-1.99$ \\
\hline \multirow[t]{2}{*}{ Other } & men & 1.57 & $1.21-2.04$ & 1.48 & $1.12-1.95$ & 1.12 & $0.91-1.38$ & 1.22 & $0.95-1.57$ & 1.62 & $1.45-1.82$ & 1.65 & $1.20-2.26$ \\
\hline & women & 1.50 & $1.16-1.92$ & 1.44 & $1.10-1.88$ & 1.29 & $1.06-1.57$ & 1.21 & $0.95-1.53$ & 1.82 & $1.62-2.04$ & 1.48 & $1.11-1.98$ \\
\hline Shaking at rest & men & 5.41 & $4.00-7.30$ & 4.64 & $3.37-6.40$ & 2.38 & $1.78-3.17$ & 3.97 & $2.93-5.39$ & 2.49 & $2.05-3.01$ & 2.21 & $1.44-3.40$ \\
\hline & women & 3.43 & $2.51-4.69$ & 3.27 & $2.35-4.53$ & 2.41 & $1.83-3.18$ & 3.63 & $2.70-4.87$ & 2.50 & $2.06-3.06$ & 1.91 & $1.28-2.86$ \\
\hline
\end{tabular}

formally assessed or treated. Further investigation is also needed of the possible contribution of high levels of manganese (commonly found in drinking water in rural Bangladesh [3]) to Parkinson-like illness [4]. If this were demonstrated, primary prevention of the disease and subsequent disability might be feasible. There is also some scope for intervention to meet the needs of the relatively small group - a total of 1243 - who reported that they would be helped by a prosthesis, mainly to aid mobility or vision. The high rate of disability reported by those with urinary incontinence is of particular interest, not least because of the possibility of intervention to improve its management $[5,6]$. The direction of causality between the incontinence and the reported disability (and the relation to depression) is likely to be complex. Given that toilet facilities in Bangladeshi village homes are outside the main living quarters, the ability to hold urine may be severely challenged in an elderly person with poor mobility and 
Table 7 Relation of total disability (log score) to health problems $(\mathrm{N}=43112)$

\begin{tabular}{lccc}
\hline & Standardised $\beta$ eta & $\mathbf{t}$ & $\mathbf{p}<$ \\
\hline Pain in joints & 0.040 & 9.47 & 0.000 \\
Chest pain & 0.058 & 13.54 & 0.000 \\
Breathing problems & 0.050 & 12.02 & 0.000 \\
Incontinence & 0.119 & 27.53 & 0.000 \\
Depression & 0.101 & 23.54 & 0.000 \\
Paralysis & 0.161 & 38.90 & 0.000 \\
Itching & 0.030 & 7.20 & 0.000 \\
Other & 0.089 & 21.93 & 0.000 \\
Shaking at rest & 0.132 & 31.80 & 0.000 \\
Sex: female & 0.062 & 13.20 & 0.000 \\
No living spouse & 0.054 & 11.23 & 0.000 \\
Age (continuous) & 0.318 & 73.23 & 0.000 \\
\hline
\end{tabular}

vision. A program to increase mobility and to improve the management of urinary incontinence would have priority in this population.

The strength of the study lies in the representation of functional difficulties and ill-health in an entire population of elderly rural villagers and in the completeness of the data: there were very few refusals and the paramedics were scrupulous about completing every question. The ability to match a substantial, and apparently representative, sub-group to census data collected 5 years earlier was also a strength of the study, allowing assessment of socio-demographic factors independent of current difficulties. The main weaknesses were the uncertainty about true age and the related difficulty of establishing a definitive list of eligible participants. Also, the data collected, both in the survey and census, failed to catch some elements of importance. While the survey asked about difficulties in understanding speech, for example, it did not ask about difficulties of expression: while the census asked about current smoking habit, it did not include amount smoked, or allow us to identify ex-smokers who had, perhaps, stopped smoking after developing disability, prior to the census. The pattern of causality was also uncertain for other observed relationships such as illiteracy and difficulty carrying heavy loads (where the physical demands may have been greater than for those with education) and the high levels of disability in those men who had already given up work by the time of the census, 5 years previously. Interpretation of the relation between poor functional capacity and reports of very often feeling depressed is also critical to decisions about interventions, designed to reduce both objective incapacity and also feelings of hopelessness. The study did not include objective measures of capacity, but relied on the villager's own report of degree of difficulty with each dimension: such self-perception of incapacity may be the appropriate metric, although perhaps less so for those with cognitive impairment. It was reassuring that the paramedics very seldom recorded that the degree of disability was under-estimated. The converse - of exaggerating disability - was not explored systematically, but the low proportions reporting 'much difficulty', particularly in those below 70 years does not suggest that exaggeration was widespread.

This is not the first study of disability in Bangladesh, although it is by far the largest, covering villages from 4 Divisions of the country. An earlier community based study of some of the same villages found that $50 \%$ of those $>80$ years had physician diagnosed disabilities, most frequently hearing, vision and movement difficulties [7]. Data from Matlab, an area to the south east of Dhaka, was included in the report of the WHO Sage studies, and showed greater disability in women, in older respondents, in people who were single, older, and less educated [1]. The study, which included some 850 subjects $\geq 70$ years, did not report the prevalence of particular disabilities. Other reports from Matlab include an attempt to better understand the value of self-reported health status in older Bangladesh villagers which found, as in the present study, that respondents were more likely to report ill health than disability [8]. The strong relation between incontinence and depression observed here has been widely reported in other populations, including elderly people in Pakistan, with the need for cleanliness in Muslim religious observance being an additional dimension [9-11].

The messages from this study are far reaching. First, at the level of primary health care providers, the results underline the urgent need for programs focusing on the elderly, to alleviate those disabilities that are amenable to intervention and to provide support and care for those with multiple disabilities. Instituting these programs will require development of training programs and health education materials, so that care of the elderly can be successfully integrated into primary health care. Such concentration on the elderly will need new funding, and cannot rely simply on the redistribution of resources away from existing programs, such as those for mothers and children. From the study reported here it is clear that funds are needed to support programs to increase mobility, enhance vision and hearing and to decrease the toll of incontinence and depression found to be so common in these elderly villagers. Alleviating these disabilities will require new approaches to care for the rural elderly, backed by demonstration projects to evaluate the viability and effectiveness of culturally appropriate interventions. Although the study reported here has shown that family support is still provided for many (though not the majority) of these elderly villagers, with rapid urbanization, and the departure of the young 
Table 8 Types of help obtained and needed (Q15 and Q16) by age (years) and sex

\begin{tabular}{|c|c|c|c|c|c|c|c|c|c|c|c|c|c|c|c|c|c|}
\hline & \multicolumn{2}{|c|}{$<60$} & \multicolumn{2}{|c|}{$60<65$} & \multicolumn{2}{|c|}{$65<70$} & \multicolumn{2}{|c|}{$70<75$} & \multicolumn{2}{|c|}{$75<80$} & \multicolumn{2}{|c|}{$80<85$} & \multicolumn{2}{|c|}{$>85$} & \multicolumn{2}{|c|}{ Total } \\
\hline & & $\mathrm{n}$ & $\%$ & $\mathrm{n}$ & $\%$ & $\mathrm{n}$ & $\%$ & $\mathrm{n}$ & $\%$ & $\mathrm{n}$ & $\%$ & $n$ & $\%$ & $\mathrm{n}$ & $\%$ & $\mathrm{n}$ & $\%$ \\
\hline \multicolumn{18}{|l|}{ Help Given } \\
\hline \multirow[t]{2}{*}{ Some help given } & men & 96 & 7.0 & 824 & 21.5 & 1113 & 22.1 & 1435 & 28.5 & 837 & 30.9 & 820 & 41.2 & 949 & 52.3 & 6074 & 27.9 \\
\hline & women & 216 & 13.1 & 1291 & 25.3 & 1351 & 24.0 & 1415 & 33.6 & 613 & 36.7 & 779 & 48.4 & 822 & 56.6 & 6487 & 30.4 \\
\hline \multirow[t]{2}{*}{ By spouse } & men & 65 & 4.7 & 466 & 12.1 & 609 & 12.1 & 710 & 14.1 & 43.9 & 16.2 & 343 & 17.2 & 324 & 17.9 & 2955 & 13.6 \\
\hline & women & 40 & 2.4 & 85 & 1.7 & 74 & 1.3 & 59 & 1.4 & 22 & 1.3 & 18 & 1.1 & 17 & 1.2 & 315 & 1.5 \\
\hline \multirow[t]{2}{*}{ By daughter-in-law } & men & 18 & 1.3 & 116 & 4.3 & 263 & 5.2 & 455 & 9.0 & 294 & 10.9 & 338 & 17.0 & 510 & 28.2 & 2044 & 9.1 \\
\hline & women & 90 & 5.4 & 721 & 14.1 & 838 & 14.9 & 877 & 20.8 & 387 & 23.1 & 496 & 30.8 & 548 & 37.7 & 3957 & 18.6 \\
\hline \multirow[t]{2}{*}{ By son } & men & 17 & 1.2 & 186 & 4.8 & 253 & 5.0 & 282 & 5.6 & 127 & 4.7 & 145 & 7.3 & 146 & 8.1 & 1156 & 5.3 \\
\hline & women & 68 & 4.1 & 345 & 6.8 & 279 & 5.0 & 278 & 6.6 & 115 & 6.9 & 115 & 7.2 & 125 & 8.6 & 1325 & 6.2 \\
\hline \multirow[t]{2}{*}{ By daughter } & men & 16 & 1.2 & 82 & 2.1 & 123 & 2.4 & 104 & 2.1 & 71 & 2.6 & 56 & 2.8 & 69 & 3.8 & 521 & 2.4 \\
\hline & women & 46 & 2.8 & 198 & 3.9 & 191 & 3.4 & 190 & 4.5 & 85 & 5.1 & 112 & 7.0 & 118 & 8.1 & 940 & 4.4 \\
\hline \multirow[t]{2}{*}{ By grandchild } & men & 0 & 0.0 & 15 & 0.4 & 14 & 0.3 & 53 & 1.1 & 25 & 0.9 & 46 & 2.3 & 66 & 3.7 & 219 & 1.0 \\
\hline & women & 6 & 0.4 & 58 & 1.1 & 91 & 1.6 & 124 & 2.9 & 65 & 3.9 & 110 & 6.8 & 124 & 8.5 & 578 & 2.7 \\
\hline \multicolumn{18}{|l|}{ Types of help } \\
\hline \multirow[t]{2}{*}{ Mobility } & men & 16 & 1.2 & 148 & 3.9 & 246 & 4.9 & 307 & 6.1 & 222 & 8.2 & 265 & 13.3 & 363 & 20.1 & 1567 & 7.2 \\
\hline & women & 39 & 2.4 & 225 & 4.4 & 330 & 5.9 & 401 & 9.5 & 203 & 12.1 & 299 & 18.6 & 371 & 25.5 & 1868 & 8.8 \\
\hline \multirow[t]{2}{*}{ Bathing } & men & 19 & 1.4 & 121 & 3.2 & 204 & 4.0 & 308 & 6.1 & 241 & 8.9 & 269 & 13.5 & 424 & 23.5 & 1586 & 7.3 \\
\hline & women & 21 & 1.3 & 163 & 3.2 & 234 & 4.2 & 373 & 8.8 & 203 & 12.1 & 294 & 18.3 & 422 & 29.0 & 1710 & 8.0 \\
\hline \multirow[t]{2}{*}{ Feeding } & men & 26 & 1.9 & 160 & 4.2 & 244 & 4.8 & 317 & 6.3 & 196 & 7.2 & 198 & 10.0 & 271 & 15.0 & 1412 & 6.5 \\
\hline & women & 41 & 2.5 & 224 & 4.4 & 258 & 4.6 & 321 & 7.6 & 143 & 8.6 & 191 & 11.9 & 258 & 17.8 & 1436 & 6.7 \\
\hline \multirow[t]{2}{*}{ Washing clothes } & men & 14 & 1.0 & 73 & 1.9 & 121 & 2.4 & 196 & 3.9 & 147 & 5.4 & 139 & 7.0 & 228 & 12.6 & 918 & 4.2 \\
\hline & women & 16 & 1.0 & 100 & 2.0 & 136 & 2.4 & 213 & 5.1 & 108 & 6.5 & 179 & 11.1 & 209 & 14.4 & 961 & 4.5 \\
\hline \multirow[t]{2}{*}{ Some help needed } & men & 620 & 45.3 & 2382 & 62.1 & 3202 & 63.5 & 3309 & 65.7 & 1832 & 66.7 & 1397 & 70.3 & 1273 & 70.4 & 14015 & 64.3 \\
\hline & women & 859 & 52.0 & 3504 & 68.7 & 3871 & 68.8 & 3002 & 71.2 & 1192 & 71.3 & 1189 & 73.9 & 1074 & 73.9 & 14691 & 68.9 \\
\hline \multicolumn{18}{|l|}{ Types of help } \\
\hline Treatment & men & 373 & 27.2 & 1578 & 41.1 & 2090 & 41.4 & 2056 & 40.9 & 1231 & 45.5 & 838 & 42.2 & 834 & 46.2 & 9000 & 41.3 \\
\hline & women & 565 & 34.2 & 2322 & 45.5 & 2424 & 43.1 & 1833 & 43.5 & 753 & 45.0 & 732 & 45.5 & 703 & 48.4 & 9332 & 43.7 \\
\hline Financial & men & 214 & 15.6 & 751 & 19.6 & 1020 & 20.2 & 1081 & 21.5 & 518 & 19.1 & 459 & 23.1 & 364 & 20.1 & 4404 & 20.2 \\
\hline & women & 241 & 14.6 & 1071 & 21.0 & 1329 & 23.6 & 1052 & 25.0 & 394 & 23.6 & 402 & 25.0 & 332 & 22.8 & 4821 & 22.6 \\
\hline Prosthesis & men & 36 & 2.6 & 101 & 2.6 & 128 & 2.5 & 163 & 3.2 & 84 & 3.1 & 70 & 3.5 & 62 & 3.4 & 644 & 3.0 \\
\hline & women & 52 & 3.1 & 111 & 2.2 & 143 & 2.5 & 131 & 3.1 & 45 & 2.7 & 58 & 3.6 & 59 & 4.1 & 599 & 2.8 \\
\hline Total N & men & 1369 & & 3836 & & 5043 & & 5033 & & 2705 & & 1988 & & 1807 & & 21781 & \\
\hline & women & 1653 & & 5102 & & 5628 & & 4215 & & 1672 & & 1608 & & 1453 & & 21331 & \\
\hline
\end{tabular}

and healthy to the cities, family structures for the care of the elderly will surely break down, as has already been shown in China $[12,13]$. Where young people leave, rural communities will be faced with the need to fill this gap with the provision of community facilities, giving help with feeding and personal care, and aids with vision and mobility to assure accessibility. With such help, the elderly can become more largely self-sufficient, as happens through comprehensive home and social care in wealthy developed counties, in which the maintenance of the elderly at home is seen as a prime goal for social programs. In Bangladesh, the government has begun to recognize the need for social welfare programs for the elderly, but the problems are still substantial, both in Bangladesh and other poor developing countries. Until recently the focus of WHO and donor agencies has been very largely on infants, children and those of reproductive age, but it is no longer defensible to assume either that the rural poor will not survive to old age - they increasingly do - or that younger women in the household will continue to be willing and available to help with basic needs. A new vision is needed in which the 
Table 9 Any help received by need (logistic regression $\mathrm{N}=$ 43112)

\begin{tabular}{lcc}
\hline & Odds Ratio & $\mathbf{9 5 \%} \mathbf{C l}$ \\
\hline Indication of Need & 1.13 & \\
No living spouse & 1.10 & $1.07-1.19$ \\
Female & 1.04 & $1.04-1.15$ \\
Age (continuous) & & $1.03-1.04$ \\
Disabilities (much difficulty) & 1 & - \\
None & 1.71 & $1.61-1.81$ \\
One & 2.83 & $2.59-3.09$ \\
Two & 4.47 & $4.03-4.96$ \\
$3-5$ & 14.94 & $12.66-17.65$ \\
6 or more & & \\
\hline
\end{tabular}

residual capacities of the old are nurtured, remediable deficiencies are attacked vigorously and community facilities put in place to reduce the physical, emotional and cognitive isolation of old people living out their years in discomfort and poverty.

\section{Conclusion}

In this study, disabled elderly residents of rural villages in Bangladesh were found to be dependent on the family for help. With family cohesiveness under threat from migration to the city, there is a pressing need for the development and critical evaluation of community-based interventions designed specifically for the elderly in poor rural societies. New approaches to training and practice will be needed to integrate such disability management into primary care.

\section{Additional file}

Additional file 1: Survey Card.

Competing interests

The authors declare that they have no competing interests.

\section{Author details}

'University of Alberta, 5-30 University Terrace, 8303-112 St, Edmonton, AB, Canada T6G 1K4. ${ }^{2}$ Community-based Medical College, Gonoshasthaya Kendra, Saver, Bangladesh. ${ }^{3}$ Gonoshasthaya Kendra, Saver, Bangladesh

${ }^{4}$ Imperial College, London, UK. ${ }^{5}$ Gonoshasthaya Kendra, Dhaka, Bangladesh.

\section{Authors' contributions}

NC, ZC and CMCD conceived the study, developed the design, methodology and measurement tools. RH and $\mathrm{MC}$ organized the field work and verification of information accuracy. NC and $M C$ were responsible for data entry and verification. NC and CMCD .planned and executed the data analysis. NC drafted the paper and acts as guarantor. All authors commented critically on the initial draft and have agreed the final text.

\section{Ethical issues}

The proposal for this project was approved by the Health Research Ethics Board of the University of Alberta (Pro00009158) and by the Gonoshasthaya Kendra ethics committee. All subjects gave oral informed consent.

\section{Sources of funding}

The work was funded from research funds held at the National Heart and Lung Institute, London.The funding source had no role in the study design, collection, analysis or interpretation, in the writing of the report or in the decision to submit.

Received: 11 March 2012 Accepted: 25 May 2012

Published: 25 May 2012

\section{References}

1. Razzaque A, Nahar L, Akter Khanam M, Kim Streatfield P: Sociodemographic differentials of adult health indicators in Matlab, Bangladesh: self-rated health, health state, quality of life and disability level. Glob Health Action 2010, Supplement 2:70-77.

2. Madans JH, Loeb ME, Altman BM: Measuring disability and monitoring the UN convention on the rights of persons with disabilities: the work of the Washington Group on disability statistics. BMC Public Health 2011, 11 ((Suppl 4):S4.

3. Arsenic contamination of groundwater in Bangladesh. In BGS Technical Report WC/00/19. Edited by Kinniburgh DG, Smedley PL. Keyworth: British Geological Survey; 2001.

4. Kondakis XG, Makris N, Leotsinidis M, Prinou M, Papapetropoulos: Health effects of high manganese concentration in drinking water. Arch Environ Health 1989, 44:175-178.

5. Hay-Smith EJ, Dumoulin C: Pelvic floor training versus no treatment, or inactive control treatments for urinary incontinence in women. Cochrane Database Syst Rev 2006, 1:CD005654.

6. Kim H, Yoshida H, Suzuki T: The effects of multidimensional exercise treatment on community-dwelling elderly Japanese women with stress, urge and mixed urinary incontinence: a randomized controlled trial. Int J Nursing Stud 2011, 48:1165-1172.

7. Hosain GMM: Disability problem in a rural area of Bangladesh. Bangladesh Med Res Counc Bull 1995, 21:24-31.

8. Rahman MO, Barsky AJ: Self-reported health among older Bangladeshis: how good a health indicator is it? Gerontologist 2003, 43:856-863.

9. Yip SK, Cardozo L: Psychological morbidity and female urinary incontinence. Best Pract Res Clin Obstet Gynaecol 2007, 21:321-329.

10. Ganatra HA, et al: Prevalence and predictors of depression among an elderly population of Pakistan. Aging Ment Health 2008, 12:349-356.

11. Wilkinson K: Pakistani women's perceptions and experiences of incontinence. Nurs Stand 2001, 16:33-39.

12. Joseph AE, Phillips DR: Ageing in rural China: impacts of increasing diversity in family and community resources. J Cross Cult Gerontol 1999, 14:153-168.

13. Giles J, Mu R: Elderly parent health and the migration decisions of adult childref: evidence from rural China. Demography 2007, 44:265-288.

doi:10.1186/1471-2458-12-379

Cite this article as: Cherry et al: Disability among elderly rural villagers: report of a survey from Gonoshasthaya Kendra, Bangladesh. BMC Public Health 2012 12:379.

\section{Submit your next manuscript to BioMed Central and take full advantage of:}

- Convenient online submission

- Thorough peer review

- No space constraints or color figure charges

- Immediate publication on acceptance

- Inclusion in PubMed, CAS, Scopus and Google Scholar

- Research which is freely available for redistribution 